\title{
Numerical Modelling of Regular Waves Propagation and Breaking Using Waves2Foam
}

\author{
B. Chenari, S. S. Saadatian, and Almerindo D. Ferreira
}

\begin{abstract}
Nowadays, ocean energy has attracted more attention among the researchers due to its massive energy potential. As designing, constructing and testing prototypes are both expensive and time consuming, recently, many researchers have developed numerical wave tanks to simulate the behaviour of the waves as well as the interaction of waves with wave energy converters. This paper aims to model numerical wave tanks, using waves2Foam - a solver within OpenFOAM, to show the propagation of waves, as well as different wave breaking types. Firstly, a flat-bottom wave tank is modelled in order to simulate both generation and absorption of the waves. Such results are benchmarked against laboratory experiments data and the comparison between simulation and experiment results showed a good agreement. Furthermore, some additional cases are modelled to assess the capability of waves2Foam in absorption of waves' reflection from the outlet boundary. Secondly, different sloped wave tanks are modelled to investigate the capability of waves2Foam in properly simulating wave breakings. Results demonstrated that waves2Foam is not only able to well simulate wave generation and absorption but it is also able to simulate all types of wave breaking. This work presents waves2Foam as powerful toolbox which can properly model waves based on different wave theories. However, some limitations of the solver were identified.
\end{abstract}

Index Terms-Breaking waves, numerical wave tank, OpenFOAM, relaxation zone, Stokes wave theory, waves 2 Foam.

\section{INTRODUCTION}

Pollutant depletion and global warming caused by consumption of fossil energies have led researchers to find sustainable alternatives. Nowadays, renewable energy has become the top priority in most developed and some developing countries. There are various types of renewable energy with different capacity all over the world. In recent years, as wave energy has the highest potential in comparison with other renewables, it attracts more attention among the researchers and research institutes. However, there are still some technical issues and barriers which are required to be solved in order to get the real wave power. It is definitely crucial to design and construct sturdy wave energy converters which can cope with the harsh condition of the oceans [1].

In the past, wave studies were based on experimental and physical models which were both time and cost consuming. Today, due to improvement and development of powerful computers and computational methods, numerical models are mostly used. Recently, many researchers have developed their

Manuscript received April 21, 2014; revised July 2, 2014.

The authors are with the University of Coimbra, Department of Mechanical Engineering, Polo II, 3030-788 Coimbra, Portugal (e-mail: behrang.chenari@student.dem.uc.pt, shivasaadatian@student.dem.uc.pt, almerindo.ferreira@dem.uc.pt). numerical wave tanks to simulate ocean waves [2]. Numerical models are still under development in order to become an appropriate alternative for laboratory experiments. Numerical models are in fact mathematical models that use some sort of numerical time-stepping procedure (in transient studies) to obtain the models' behaviour over time. Numerical modelling is a powerful method of predicting and visualizing the dynamic behaviour of physical systems. Computational fluid dynamics (CFD) software uses simplified equations but they will speed up the research as well as making it easier to study behaviour of wave and its behaviour when interacting with floating devices, sloped sea bed and also shore-based devices.

Open Field Operation And Manipulation, known as OpenFOAM, is an open source computational fluid dynamics package of $\mathrm{C}++$ libraries and codes that are created to conduct numerical modelling of solid and fluid mechanics problems and it was first released in 2004 [3]. OpenFOAM is distributed with a large number of solvers and utilities to cover a wide range of problems. However, it is possible for users to write their own codes and solvers for their specific problems or to modify the existing solvers due to the open source nature of OpenFOAM. Waves2Foam is a toolbox recently developed by OpenFOAM users to simulate free surface wave generation and absorption [4]. A relaxation zone technique known as active sponge layer has been applied to the library as well as a large range of different wave theories. The base of this toolbox is interFoam while an active sponge layer zone defined as relaxation zone method has been added to the solver.

\section{GOVERNING EQUATIONS AND WAVE THEORIES}

There are various types of wave theories, as [5] introduced and explained them but Navier-Stokes equations are basically used by OpenFOAM which are described as below:

$$
\begin{aligned}
& P\left(\frac{\partial u}{\partial t}+u \frac{\partial u}{\partial x}+v \frac{\partial u}{\partial y}+w \frac{\partial u}{\partial z}\right)=-\frac{\partial p}{\partial x}+\mu\left(\frac{\partial^{2} u}{\partial x^{2}}+\frac{\partial^{2} u}{\partial y^{2}}+\frac{\partial^{2} u}{\partial z^{2}}\right)+p g_{x} \\
& \rho\left(\frac{\partial v}{\partial t}+u \frac{\partial v}{\partial x}+v \frac{\partial v}{\partial y}+w \frac{\partial v}{\partial z}\right)=-\frac{\partial p}{\partial y}+\mu\left(\frac{\partial^{2} v}{\partial x^{2}}+\frac{\partial^{2} v}{\partial y^{2}}+\frac{\partial^{2} v}{\partial z^{2}}\right)+\rho g_{y} \\
& \rho\left(\frac{\partial w}{\partial t}+u \frac{\partial w}{\partial x}+v \frac{\partial w}{\partial y}+w \frac{\partial w}{\partial z}\right)=-\frac{\partial w}{\partial z}+\mu\left(\frac{\partial^{2} w}{\partial x^{2}}+\frac{\partial^{2} w}{\partial y^{2}}+\frac{\partial^{2} w}{\partial z^{2}}\right)+\rho g_{z}
\end{aligned}
$$

where $\rho$ represents density in [kg.m-3], t represents time in $[\mathrm{s}], p$ represents pressure in $[\mathrm{Pa}], g$ represents acceleration 
gravity in [m.s-2], $\mu$ represents dynamic viscosity in [Pa.s] and $u, v$ and $w$ represent velocity components in $x, y$ and $z$ directions, respectively in [m.s-1].

As the flow is considered incompressible, $\rho$ is constant so the continuity equation that must be satisfied is as below:

$$
\frac{\partial u}{\partial x}+\frac{\partial v}{\partial y}+\frac{\partial w}{\partial z}=0
$$

Volume of fluid (VOF) is a numerical method for tracking and locating free surface which is the interface of air and water in the present study. This method is used by OpenFOAM to specify the fraction of each fluid (air and water) in each cell. The volume (phase) fraction equation is presented below in which $\alpha$ represents the volume (phase) fraction, $t$ represents time and $\mathrm{U}$ refers to velocity. $\alpha$ is always between 0 and 1. $\alpha=0$ means the cell is fully filled by air and $\alpha=1$ means the cell is only filled by water.

$$
\frac{\partial \alpha}{\partial t}+\nabla \cdot(\alpha U)=0
$$

The density of each cell can be calculated by the following equation, where $\rho_{w}$ is the water density and $\rho_{a}$ is the air density:

$$
\rho=\alpha \rho_{w}+(1-\alpha) \rho_{a}
$$

Note that this density is the density of the mixture of air and water inside each cell.

Among all wave theories, Stokes wave theory has been often applied to the studies which investigating behaviour of waves. Stokes first order wave or airy wave theory refers to a linear wave theory which is used for modelling of gravity waves on the surface of a fluid. Stokes first order wave theory is used in coastal and ocean engineering for simulating waves' behaviour. This theory is also used for simulating tsunami waves before reaching the coastal area. Stokes second order wave theory refers to a non-linear theory which is used for modelling of periodic regular free surface waves. Stokes second order wave theory is generally used for simulation of the interaction between waves and structures (both shore-based and off-shore). They are applied on the studies in order to specify wave behaviours such as free surface elevation and flow particle velocity. As Stokes theory does not work well for shallow water, it is mostly used for deep water and medium depth areas.

\section{A. Particle Velocity Equation}

Particle velocity is the velocity of a particle which is transferred by a wave. The particle velocity according the Stokes second order theory is given as below:

$$
\begin{aligned}
& u=-\frac{\partial \varphi}{\partial x}=\frac{H}{2} \frac{g h}{w} \frac{\cosh k(h+y)}{\cosh k h} \cos (k x-w t) \\
& +\frac{3}{16} \frac{H^{2} w k \cosh 2 k(h+y)}{\sinh ^{4} k h} \cos 2(k x-w t)
\end{aligned}
$$

$$
\begin{aligned}
& v=-\frac{\partial \varphi}{\partial y}=\frac{H}{2} \frac{g k}{\omega} \frac{\sinh k(h+y)}{\cosh k h} \sin (k x-\omega t) \\
& +\frac{3}{16} \frac{H^{2} \omega k \sinh 2 k(h+y)}{\sinh ^{4} k h} \sin 2(k x-\omega t)
\end{aligned}
$$

$u$ is the horizontal component of particle velocity and $v$ is the vertical component. Both $u$ and $v$ are partial derivatives of velocity potential $\varphi . H$ represents the wave height from peak to trough in $[\mathrm{m}], g$ represents acceleration due to gravity in $\left[\mathrm{m} . \mathrm{s}^{-2}\right], h$ represents water depth in $[\mathrm{m}]$ while $y$ is the vertical coordinate to describe wave motion (the points in which $y=0$ makes a line known as still water level). $t$ represents time in [s], $x$ represents the distance along longitudinal direction in $[\mathrm{m}]$, $\omega$ represents the frequency of the wave in $\left[\operatorname{Rad} . \mathrm{s}^{-1}\right]$ and $k$ represents the wave number in $\left[\operatorname{Rad} . \mathrm{m}^{-1}\right] . \omega$ and $k$ are defined in following equations in which $L$ is the wavelength in [m].

$$
\begin{gathered}
\omega=(g k \tanh k h)^{1 / 2} \\
k=\frac{2 \pi}{L}
\end{gathered}
$$

\section{B. Surface Elevation Equation}

In waves based on Stokes second order theory, the following equation, known as surface elevation equation, shows the displacement of water surface from still water level (SWL). The result of the numerical model developed in present study is validated against the analytical result of surface elevation equation.

$$
\eta=\frac{H}{2} \cos (k x-w t)+\frac{H^{2} k}{16} \frac{\cosh k h}{\sinh ^{3} k h}(2+\cosh 2 k h) \cos 2(k x-w t)(11)
$$

\section{BREAKING WAVES AND SURF ZONE}

A breaking wave is a water surface wave that its amplitude reaches a critical point which causes the crest of wave breaks and comes down. Waves will break both in shallow and deep water but the reason of breaking of wave in shallow water and deep water is completely different. In shallow water breaking of the waves is due to reaching shallower area (the beach) and also because wave heights are greater than before in this region while in deep water, breaking of waves is because of hydrodynamic instability [6].

The region near the beach where wave breaks is called surf zone. The study will show that OpenFOAM has the ability to model the waves in surf zone considering a parameter called surf similarity, which can specify the type of breaking waves as well as wave run-up. As pointed out in [7], there are four types of breaking waves. Three types of breaking waves are listed and briefly explained in following.

\section{A. Spilling Waves}

When the sea bed has a soft slope approaching the beach, the wave breaking type would be spilling. Spilling waves breaking time is quite longer than other breaking types. Fig. 1 shows propagation of the spilling wave. 


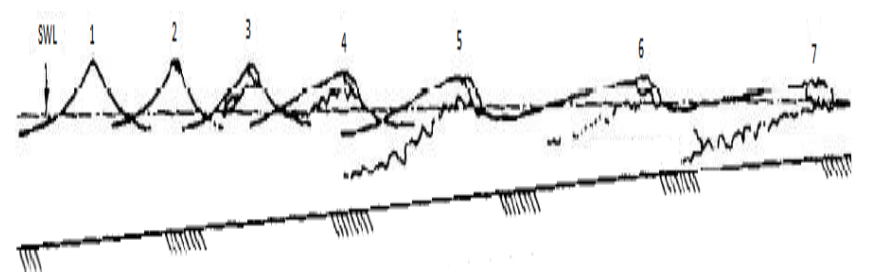

Fig. 1. Spilling wave breaking (numbers present the steps of breaking), (Source: [8]).

\section{B. Plunging Waves}

When the sea bed has steeper slope or a sudden depth changes approaching the beach, the crest of waves curls and breaks down. This type of breaking is called plunging. Plunging waves generally break with more energy comparing with spilling waves. Fig. 2 shows propagation of the plunging wave.

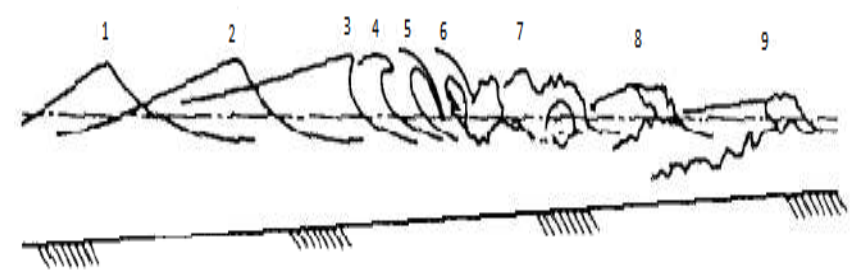

Fig. 2. Plunging wave breaking (numbers present the steps of breaking), (Source: [8]).

\section{Surging Waves}

When the sea bed has a very steep slope approaching the beach the crest of the wave keep going without breaking and finally a small breaking will happen with a little foams and bubbles. This type of breaking usually occurs in areas with a narrow surf zone. Fig. 3 shows propagation of the surging wave.

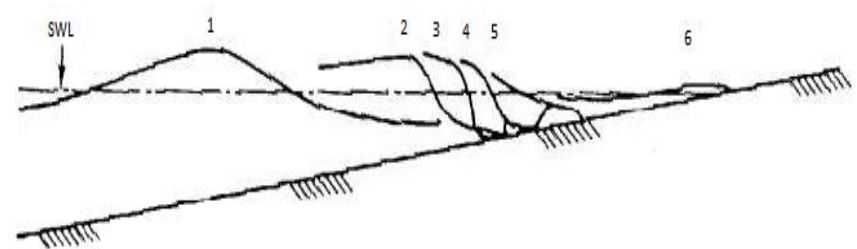

Fig. 3. Surging wave breaking (numbers present the steps of breaking), (Source: [8]).

\section{Surf Similarity}

The Iribarren number or the surf similarity number is a dimensionless number for specifying the type of gravity wave breaking approaching to surf zone and sloping beach [9]. This parameter was first introduced by those authors and the number is defined as the following equation:

$$
\xi=\frac{\tan \beta}{\sqrt{\frac{H}{L_{0}}}}
$$

Also $L_{0}$, deep water wave length, can be defined as below:

$$
L_{0}=\frac{g}{2 \pi} T^{2}
$$

By integrating (12) and (13), the surf similarity number can be defined as followings:

$$
\xi_{0}=\frac{\tan \beta}{\sqrt{\frac{2 \pi H_{0}}{g T^{2}}}}
$$

where $\xi_{0}$ represents surf similarity number for deep water region, $\beta$ represents the bed slope angle in [degree], $H_{0}$ represents the deep water wave height in $[\mathrm{m}], L_{0}$ represents the deep water wave length in [m], $T$ represents period in [s] and $g$ is gravity acceleration in $\left[\mathrm{m} \cdot \mathrm{s}^{-2}\right]$.

Table I shows different types of breaking waves using surf similarity number which were presented by [10].

\begin{tabular}{ll}
$\begin{array}{l}\text { TABLE I: SURF SIMILARITY APPROXIMATION FOR DISTINGUISHING } \\
\text { BREAKING TYPE [10] }\end{array}$ \\
\begin{tabular}{ll} 
Breaking Type & $\xi_{0}$ \\
\hline Surging or Collapsing & $\xi_{0}>3.3$ \\
Plunging & $0.5<\xi_{0}<3.3$ \\
Spilling & $\xi_{0}<0.5$ \\
\hline
\end{tabular} \\
\hline
\end{tabular}

\section{Methodology}

\section{A. Definition of Modelled Scenarios}

In this study a basic flat bottom numerical wave tank is simulated to replicate the propagation of ocean waves, and also sloped numerical wave tanks are simulated to show different wave breaking types.

\section{1) Basic flat-bottom numerical wave tank}

Fig. 4 shows a schematic view of the first scenario geometry.

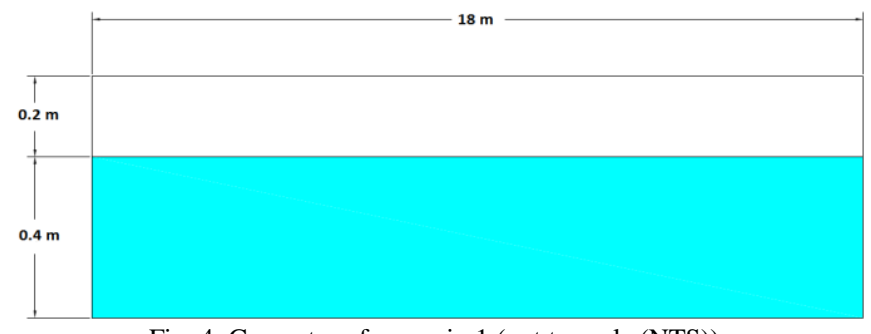

Fig. 4. Geometry of scenario 1 (not to scale (NTS)).

\section{2) Sloped numerical wave tank}

This scenario is implemented for simulation of breaking waves. To do so, this scenario is divided to three different models where each one simulates one type of wave breaking. Scenario $2 \mathrm{~A}$ intends to simulate a spilling wave breaking, scenario $2 \mathrm{~B}$ simulates a plunging wave breaking and in scenario $2 \mathrm{C}$ a surging wave breaking is simulated. As discussed before, the breaking type depends on the slope of sea bed, so different slopes are considered for scenarios $2 \mathrm{~A}$, $2 \mathrm{~B}$ and $2 \mathrm{C}$ which are indicated by $\beta_{1}, \beta_{2}$, and $\beta_{3}$ respectively. Geometries of this scenario are shown in Fig. 5, Fig. 6 and Fig. 7 , sequentially. 


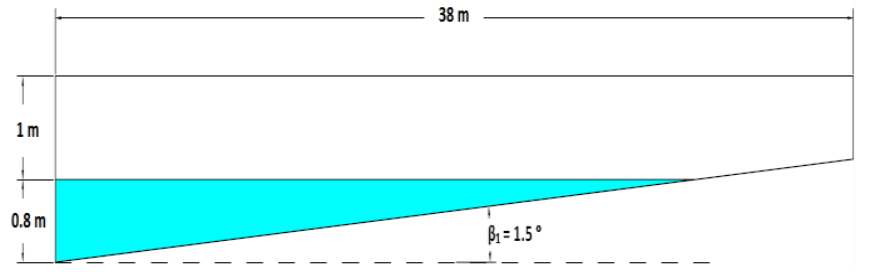

Fig. 5. Geometry of scenario $2 \mathrm{~A}, \beta_{1}=1.5^{\circ}(\mathrm{NTS})$.

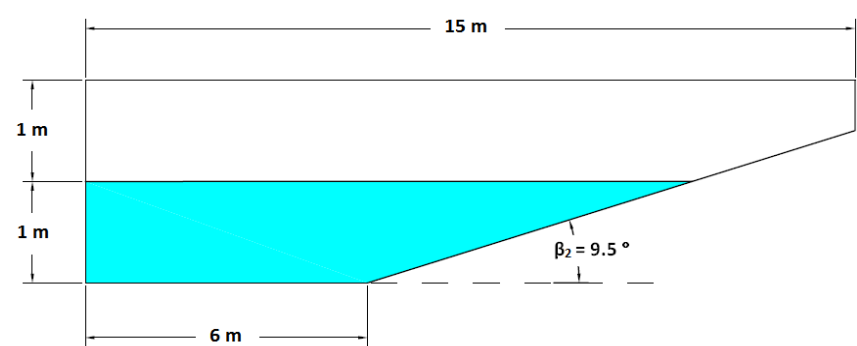

Fig. 6. Geometry of scenario $2 \mathrm{~B}, \beta_{2}=9.5^{\circ}(\mathrm{NTS})$.

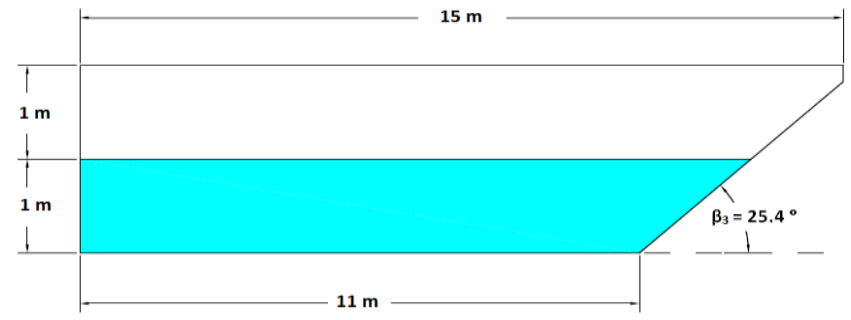

Fig. 7. Geometry of scenario $2 \mathrm{C}, \beta_{3}=25.4^{\circ}(\mathrm{NTS})$.

\section{B. Mesh Generation}

For this master thesis, the BlockMesh utility is used for generating meshes for both scenarios. Table II shows the mesh size of each scenario.

TABLE II: MESH SIZE FOR EACH SCENARIO.

\begin{tabular}{ccc} 
& \multicolumn{2}{c}{ TABLE II: MESH SIZE FOR EACH SCENARIO. } \\
\hline \multirow{2}{*}{ Scenario } & $\begin{array}{c}\text { Cell size in } \boldsymbol{x} \text { direction } \\
{[\mathbf{m}]}\end{array}$ & \begin{tabular}{cc} 
Cell size in $\boldsymbol{y}$ direction $[\mathbf{m}]$ \\
\hline 1
\end{tabular} \\
\hline 2A & 0.05 & 0.01 \\
2B & 0.04 & 0.01 \\
2C & 0.04 & 0.02 \\
\hline
\end{tabular}

\section{Input Data}

This section presents all data used as inputs for the simulation processes.

\section{1) Wave characteristics}

The characteristics of waves used in scenarios are presented in Table III.

\begin{tabular}{cccccc}
\multicolumn{7}{c}{ TABLE III: WAVE PARAMETERS FOR EACH SCENARIO } \\
\hline $\begin{array}{c}\text { Scena- } \\
\text { rio }\end{array}$ & $\begin{array}{c}\text { Wave } \\
\text { Length } \\
(\boldsymbol{L})[\mathbf{m}]\end{array}$ & $\begin{array}{c}\text { Wave } \\
\text { Height }(\boldsymbol{H})\end{array}$ & $\begin{array}{c}\text { Steepn } \\
\text { ess }\end{array}$ & $\begin{array}{c}\text { Period } \\
(\boldsymbol{T})[\mathbf{s}]\end{array}$ & $\begin{array}{c}\text { Water } \\
\text { Depth } \\
(\boldsymbol{H})[\mathbf{H})\end{array}$ \\
\hline 1 & 3.695 & 0.1 & 0.027 & 2 & 0.4 \\
2A & 5 & 0.2 & 0.04 & 2.05 & 0.8 \\
2B & 5 & 0.2 & 0.04 & 1.94 & 1 \\
2C & 5 & 0.1 & 0.02 & 1.94 & 1 \\
\hline
\end{tabular}

\section{2) Physical properties}

The physical properties that are used in this study are presented in Table IV.
TABLE IV: PHYSICAL PROPERTIES

\begin{tabular}{|c|c|c|}
\hline Property & & Value \\
\hline Acceleration gravity $(g)\left[\mathrm{m} \cdot \mathrm{s}^{-2}\right]$ & & 9.81 \\
\hline \multirow{2}{*}{ Density $(\rho)\left[\mathrm{kg} \cdot \mathrm{m}^{-3}\right]$} & Water & 1000 \\
\hline & Air & 1.2 \\
\hline \multirow{2}{*}{ Kinematic Viscosity $(\mu)\left[\mathrm{m}^{2} \cdot \mathrm{s}^{-1}\right]$} & Water & $10^{-6}$ \\
\hline & Air & $1.48 \times 10^{-5}$ \\
\hline Surface Tension $(\sigma)\left[\mathrm{N} . \mathrm{m}^{-1}\right]$ & & 0.07 \\
\hline
\end{tabular}

\section{Boundary Condition}

Table V summarize the boundary conditions applied to each boundary in all fields.

TABLE V: SUMMARY OF BOUNDARY CONDITIONS

\begin{tabular}{lccc} 
Boundary & alpha1 & P-rgh & U \\
\hline inlet & waveAlpha & zeroGradient & waveVelocity \\
outlet & $\begin{array}{c}\text { zeroGradie } \\
\text { nt }\end{array}$ & zeroGradient & fixedValue \\
& $\begin{array}{c}\text { zeroGradie } \\
\text { nt }\end{array}$ & zeroGradient & fixedValue \\
bottom & inletOutlet & totalPressure & pressureInletOutlet \\
& & & Velocity \\
atmosphere & empty & empty & empty \\
& & & \\
\hline
\end{tabular}

\section{RESUlT AND DisCUSSION}

\section{A. Scenario 1: Basic Flat-Bottom Numerical Wave Tank}

Fig. 8 and Fig. 9 Show that waves2Foam simulates propagation of a regular wave within a basic numerical wave tank. The results are already validated against theoretical and experimental data and have showed good agreements. Using waves2Foam, it is also possible to simulate waves based on different wave theories as they are pre-defined in the solver library.

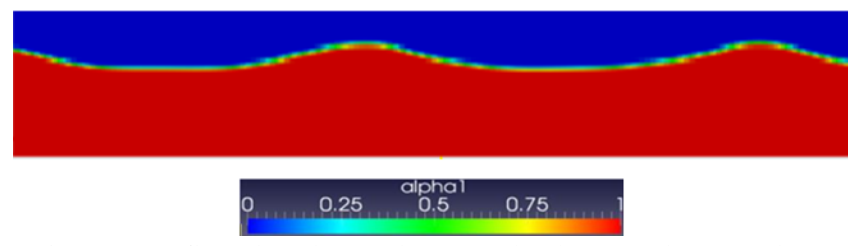

Fig. 8. Wave flume based on Stokes second order wave theory, Volume Fraction (alpha1) field at $t=16 \mathrm{~s}$ (propagation direction is from left to right).

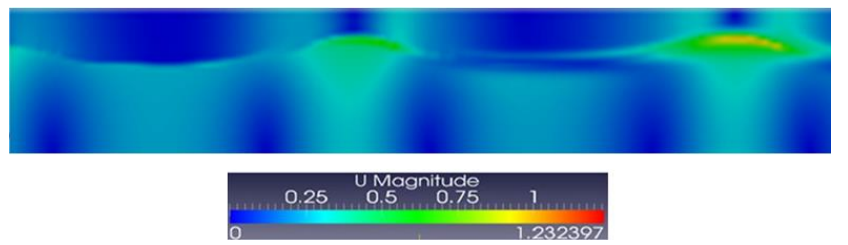

Fig. 9. Wave flume based on Stokes second order wave theory, Velocity (U) field at $t=16 \mathrm{~s}$ (propagation direction is from left to right).

This solver is validated against the laboratory experiment conducted by [11]. The numerical model (bejiBattjes tutorial) was originally performed by toolbox developer [12], who 
validates the simulation result against experimental data. As reported by many studies such as [13], [14] and [6], validation results of present study also show agreement between simulation and experiment results in the gauges before the submerged bar while the disagreements start from gauges 7 and 8 and increase in gauges 9 to 11 . These disagreements are mainly due to development of higher harmonics that occurs after the submerged bar.

Furthermore, in order to show the importance of implementing relaxation method that actually works like an active sponge layer to prevent wave reflection at the outlet boundary, we removed the relaxation zone from the scenario one. The results depicted in Fig. 10 shows the comparison between the wave flume with implemented relaxation zone and the one in which the relaxation zone is removed.

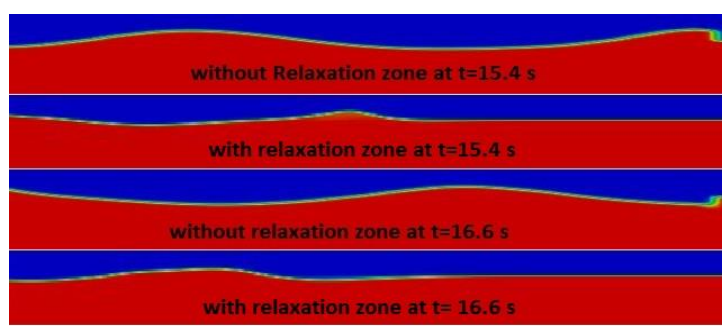

Fig. 10. Influence of implementing relaxation zone at the outlet boundary of wave flume (propagation direction is from left to right).

Fig. 10 shows only a focused part of the end of the wave flume in which the influence of relaxation zone is depicted. As shown, the relaxation zone causes absorption of the waves at the outlet boundary and prevents reflection of the wave while the one in which relaxation zone is removed apparently shows the reflection. This reflection influences the study and causes lots of mistakes in simulation. Therefore, it can be concluded that, relaxation zone method makes numerical simulation of waves more precise by preventing reflection of the waves from outlet boundary.

\section{B. Scenario 2: Sloped Numerical Wave Tank}

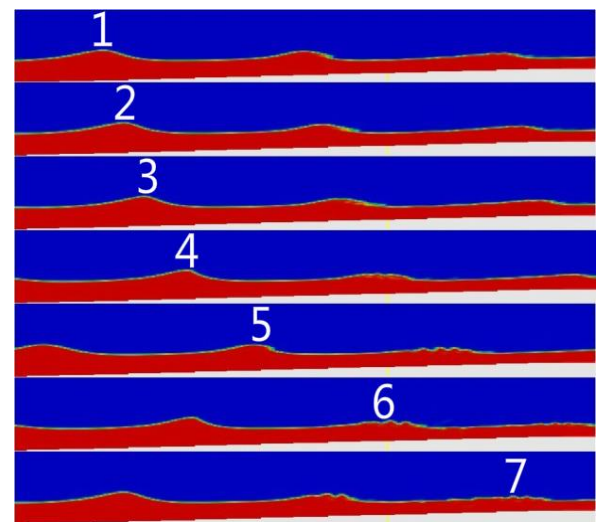

Fig. 11. Spilling wave breaking simulation (scenario 2A).

This scenario is developed in order to present the capability of waves2Foam in simulating different wave breaking types. As mentioned before, three different cases are defined in order to simulate different types of breaking waves. The results show that waves 2 Foam is able to properly simulate different wave breaking types. Note that the numbers indicate the steps of breaking. Fig. 11, Fig. 12 and Fig. 13 show the result of this scenario.

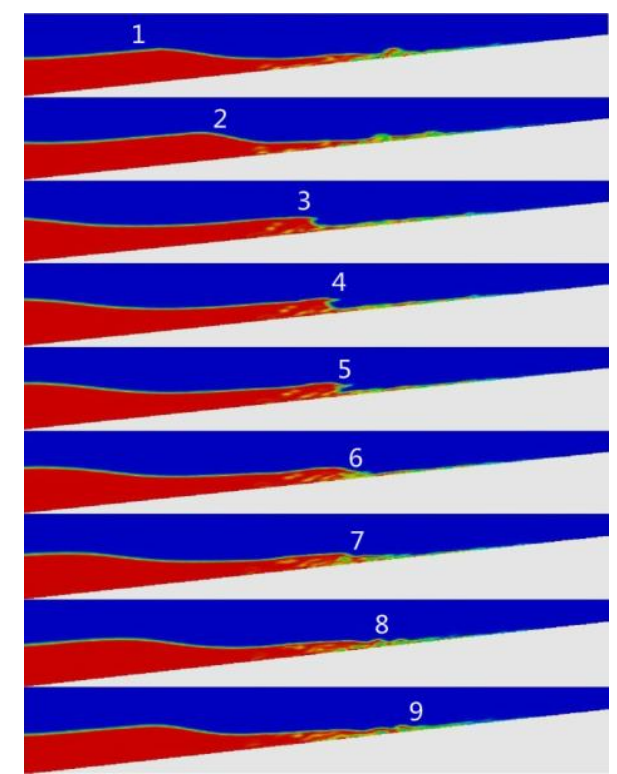

Fig. 12. Plunging wave breaking simulation (scenario 2B).

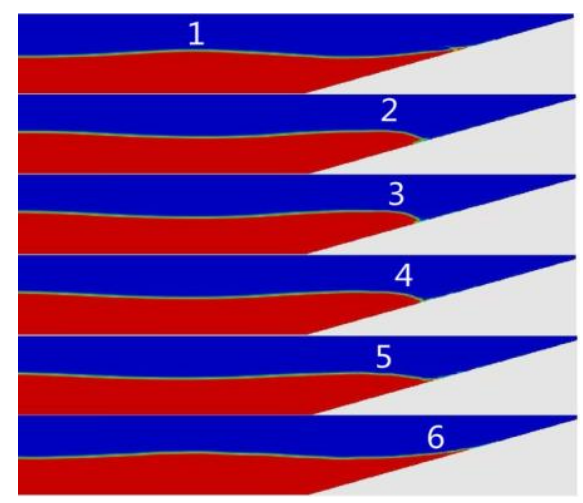

Fig. 13. Surging wave breaking simulation (scenario 2C).

\section{CONCLUSION}

In the first scenario, a basic flat-bottom wave tank was simulated to assess the capability of waves2Foam in generating and absorbing the wave and in the second scenario, sloped wave tanks was simulated to evaluate the capability of this solver in simulation of different wave breaking types. Both scenarios showed the high capacity of waves2Foam in modelling ocean waves.

To sum up, it should be noted that this study presents OpenFOAM as powerful software which can be used in ocean engineering science. Especially, due to its open source nature, OpenFOAM needs to be further developed by modifying the pre-existed solvers and codes. Moreover, present work shows that waves2Foam has the capability of correctly modelling of regular wave propagation both in generation and absorption of the waves as well as simulating the wave behaviour interacting with seabed and its breaking steps.

Finally, based on limitation of the software and toolbox discussed in this work, some recommendations for future developments are presented below:

1) Reasons which cause higher harmonics after the submerged bar such as non-linearity and dispersion should be investigated to find a proper way, preventing higher harmonics after the bar in order to improve the agreement between results of simulations and laboratory experiments; 
2) The ability of the software and toolbox in terms of simulating waves with steepness above 0.05 should be assessed and increased;

3) As an extension of the present work, also the ability of waves2Foam in simulating floating object within the numerical wave tank should be checked.

\section{REFERENCES}

[1] Q. Du and D. Y.C. Leung, "2D numerical simulation of ocean waves," Marine and Ocean Technology, vol. 9, p. 2183, 2011.

[2] P. F. Liu and I. Losada, "Wave propagation modeling in coastal engineering," Journal of Hydraulic Research, vol. 40, no. 3, pp. 229-240, 2002.

[3] OpenFOAM. (2013). User guide version 2.2.0. [Online]. Available: http://www.openfoam.org/docs/user.

[4] N. G. Jacobsen, D. R. Fuhrman, and J. Fredsøe, "A wave generation toolbox for the open-source CFD library: OpenFoam," International Journal for Numerical Methods in Fluids, vol. 70, no. 9, pp. 1073-1088, 2012.

[5] J. D. Fenton, "Nonlinear wave theories," in The Sea, Ocean Engineering Science, B. Le Méhauté and D. M. Hanes, Eds. Wiley, New York, 1990, vol. 9, no. 1.

[6] R. J. Lambert, "Development of numerical wave tank using OpenFOAM," Ms Thesis, Energy for Sustainability Program, University of Coimbra, 2012.

[7] T. Sarpkaya and M. Isaacson, Mechanics of Wave Forces on Offshore Structures, New York: Van Nostrand Reinhold Company, 1981, vol. 96.

[8] J. E. Richardson, "FSI-96-00-TN44 Surf similarity," 1996.

[9] C. R. Iribarren and C. Nogales,. "Protection des Ports," in Proc. XVIIth International Navigation Congress, Lisbon, Portugal, 1949, pp. 31-80.

[10] J. A. Battjes, "Surf similarity," in Proc. the $14^{\text {th }}$ Internatinal Conference on Coastal Engineering, Copenhagen, Denmark, 1974, pp. 466-480.

[11] S. Beji and J. A. Battjes, "Experimental investigation of wave propagation over a bar," Coastal Engineering, vol. 19, pp. 151-162, 1993.

[12] N. G. Jacobsen. (2012). Waves2Foam toolbox bejibattjes validation case tutorial. [Online]. Available: http://openfoamwiki.net/index.php/Contrib/waves2Foam.
[13] F. Chazel, M. Benoit, and A. Ern, "Validation of a double-layer Boussinesq-type dodel for highly nonlinear and dispersive waves," in Proc. the $32^{\text {nd }}$ Internatinal Conference on Coastal Engineering, Shanghai, China, 2010, pp. 1-8.

[14] G. C. J. Morgan, J. Zang, D. Greaves, A. Heath, C. D. Whitlow, and J. R. Young, "Using the rasInterFoam CFD model for wave transformation and coastal modeling," in Proc. 32nd Conference on Coastal Engineering, Shanghai, China, 2010, pp. 1-9.

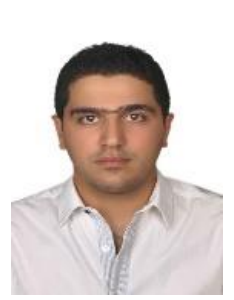

Behrang Chenari is a $\mathrm{PhD}$ student in Sustainable Energy Systems MIT-Portugal Program at University of Coimbra, he was born in 1986 in Iran. Behrang graduated from Azad University of Tehran in mechanical engineering and worked more than 4 years in Daryapalaenergy Oil \& Gas Company. He joined Energy for Sustainability Master Program at University of Coimbra in September 2012. As his master thesis, he worked numerical modeling of waves using OpenFOAM. Now, he is conducting research about developing hybrid ventilation in buildings.

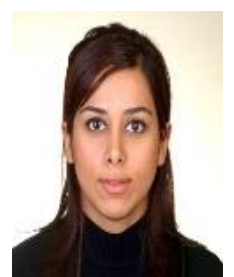

Shiva Saadatian was born in 1985 in Tehran, Iran. She received her first degree in industrial engineering from Azad University of Tehran, Iran in 2009. After her five years studying in industrial engineering, she worked as a project planning expert at M.F.S Co. in Tehran for about three years. Furthermore, she received her master degree in energy for sustainability at the University of Coimbra, Portugal in February 2014. And now she starts her PhD study in sustainable energy systems at MIT-Portugal Program at University of Coimbra.

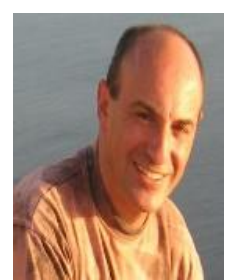

Almerindo Domingues Ferreira was born on October 21, 1964. He received his degree in mechanical engineering in 1988 from University of Coimbra. He is an assistant professor in Mechanical Engineering Department at University of Coimbra since 1999. 